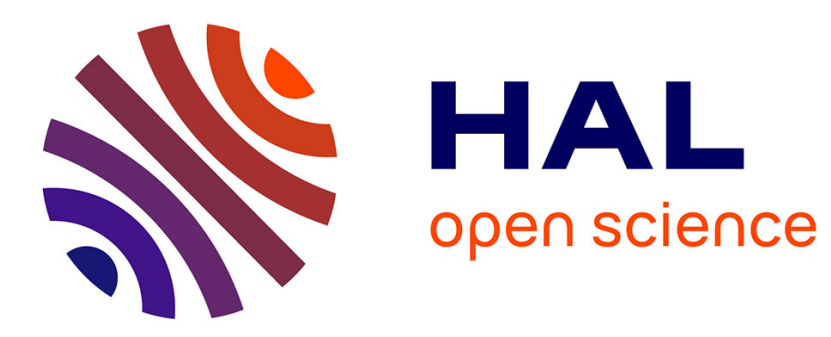

\title{
Contributions to output controllability for Linear Time Varying systems
}

Baparou Danhane, Jérôme Lohéac, Marc Jungers

\section{To cite this version:}

Baparou Danhane, Jérôme Lohéac, Marc Jungers. Contributions to output controllability for Linear Time Varying systems. IEEE Control Systems Letters, 2021, 6, pp.1064-1069. 10.1109/LCSYS.2021.3089642 . hal-03268677

\section{HAL Id: hal-03268677 https://hal.science/hal-03268677}

Submitted on 23 Jun 2021

HAL is a multi-disciplinary open access archive for the deposit and dissemination of scientific research documents, whether they are published or not. The documents may come from teaching and research institutions in France or abroad, or from public or private research centers.
L'archive ouverte pluridisciplinaire HAL, est destinée au dépôt et à la diffusion de documents scientifiques de niveau recherche, publiés ou non, émanant des établissements d'enseignement et de recherche français ou étrangers, des laboratoires publics ou privés. 


\title{
Contributions to output controllability for Linear Time Varying systems
}

\author{
Baparou Danhane, Jérôme Lohéac, and Marc Jungers *
}

June 23, 2021

\begin{abstract}
The purpose of this paper is to provide some contributions to one notion of Output controllability for Linear Time Varying (LTV) systems which is the Complete State to Output Controllability (CSOC), notion introduced in the 60 s by P. E. Sarachik and G. M. Kranc. More precisely, we consider LTV systems with direct transmission of the input to the output and establish criteria to ensure the CSOC in finite time of these systems. We also give, under the assumption of CSOC in finite time, an explicit expression of a continuous control built by means of a Gramian matrix.
\end{abstract}

Keywords: Automatic control, Open loop systems

\section{Introduction}

The Complete State to Output Controllability (CSOC) for Linear Time Varying (LTV) systems was introduced in [7] by P. E. Sarachik and G. M. Kranc in 1963. They considered LTV systems without direct transmission of the input to the output and extended the Kalman state controllability Gramian criterion to the case of output. In [6], E. Kreindler and P. E. Sarachik extended this criterion to LTV systems with direct transmission from the input to the output. For this notion, the aim is to study if one can drive in finite time the output of these systems to a prescribed value from any initially given state data. Another notion of output controllability for LTV systems without direct transmission of the input to the output, which is Complete Output Controllability can be found in [3. For this second notion, as stated in [3, Definition 4.2], the question is whether it is possible or not to steer the output from any initial output value to a prescribed output value in finite time. Note that even if the criteria given in [3] are the same as those given in [6], these two notions are rigorously distinct as it was discussed in [4, Section 6] for Linear Time-Invariant systems. So, the criteria

\footnotetext{
${ }^{*}$ Université de Lorraine, CNRS, CRAN, F-54000 Nancy, France (e-mails: \{baparou.danhane, jerome.loheac, marc.jungers $\}$ Quniv-lorraine.fr)
} 
given in [3] have to be understood in the sense of [6, Definition 3]. The authors, in [3, also gave, under some regularity assumptions, a sufficient condition of Kalman rank type to ensure the complete state to output controllability in finite time of the considered systems. To our knowledge, these are the two existing results that allow us to verify the CSOC property of LTV systems. The Gramian condition being usually difficult to check in practice, it is natural to look for some other testable controllability conditions. This has already been considered in [3. Section 4.1.3] but assuming some strong regularity conditions on the system. Moreover, for CSOC system with direct transmission from the input to the output, it could be of practical importance to have a smooth output trajectory, that is to say to have a smooth control that enables us to achieve the desired transfer.

In this paper, we consider the same system as in [6, and introduce the notion of State to Output Controllability (SOC) in finite time. This notion is actually [6], Definition 3], with $t_{0}=0$. Note however that the arguments used in the present paper remain unchanged, when $t_{0} \neq 0$. In Section 2 we recall some results on differential equations, and define the SOC notion. We also give in Problems 1 and 2 a precise formulation of the issues tackled in this paper. In Section 3. the main results are provided. In this section, we proposed Theorem 2 which gives necessary and sufficient condition for SOC in finite time. These conditions being difficult to check in practice because requiring the computation of the resolvent and the invertibility of matrices, we proposed in Theorem 3 a sufficient condition which does not involve the resolvent matrix and is easily checkable. This condition turns out to be necessary under analytic assumption. However, if the resolvent matrix can be easily computed, we also give sufficient and necessary conditions through Proposition 5 and Theorems 6 and 7 . We end Section 3 with Theorem 8 which gives an explicit expression of continuous control. These results are illustrated on an example in Section 4 and proven in Section 5. Conclusion is given in Section 6 .

The following notations are used. The sets $\mathbb{N}$ and $\mathbb{R}$ are respectively the set of nonnegative integers and of real numbers. We also define $\mathbb{R}_{+}=[0, \infty), \mathbb{N}^{*}=$ $\mathbb{N} \backslash\{0\}$ and for $k \in \mathbb{N}^{*}, \mathbb{N}_{\leqslant k}=\{0, \ldots, k\}, \mathbb{N}_{\leqslant k}^{*}=\mathbb{N}_{\leqslant k} \backslash\{0\}$ and $\mathbb{N}_{<k}=\mathbb{N}_{\leqslant k-1}$. We also set $\mathbb{N}_{\leqslant \infty}=\mathbb{N}$. Given $k, l \in \mathbb{N}^{*}, \mathbb{R}_{k}^{l}$ (with shortcut $\mathbb{R}_{1}^{l}=\mathbb{R}^{l}$ ) is the set of $l \times k$ matrices with coefficients in $\mathbb{R}$, and $0_{k}^{l}$ is its null element. For $F \in \mathbb{R}_{k}^{l}$, $\operatorname{rk} F, \operatorname{Im} F$ and $F^{\top}$ denote respectively the rank, image and transpose of $F$. Given $e_{1}, \ldots, e_{k} \in \mathbb{R}^{l}, \operatorname{Span}\left\{e_{1}, \ldots, e_{k}\right\} \subset \mathbb{R}^{l}$ is the vector space generated by $e_{1}, \ldots, e_{k}$. The Euclidean norm and product on $\mathbb{R}^{l}$ are denoted by $|\cdot|$ and $\langle\cdot, \cdot\rangle$. If $G$ is also a matrix with $l$ lines $(F \mid G)$ is the concatenation in row of the matrices $F$ and $G$. If $\left(F_{i}\right)_{i \in \mathbb{N}}$ is a sequence of matrices having $l \operatorname{rows}, \operatorname{rk}\left(F_{i}, i \in \mathbb{N}\right)$ is the dimension of $\sum_{i \in \mathbb{N}} \operatorname{Im}\left(F_{i}\right)$. For $I$ a real interval, $\mathcal{C}^{k}(I)$ stands for the set of $k$ times differentiable real functions defined on $I$ and for $g \in \mathcal{C}^{k}(I), g^{(k)}$ is the $k$-th derivative of $g$. For $T>0$, we define the Banach space $L^{\infty}(0, T)$ (respectively $L^{2}(0, T)$ ) of essentially bounded (respectively square integrable) real functions defined on $(0, T)$. We also set $H^{1}(0, T)=\left\{f \in L^{2}(0, T) \quad \mid \dot{f} \in L^{2}(0, T)\right\}$, where $\dot{f}$ is the derivative of $f$, in the sense of distributions. Finally, $L_{\mathrm{loc}}^{\infty}\left(\mathbb{R}_{+}\right)$ 
is the set of real functions defined on $\mathbb{R}_{+}$that are essentially bounded on any compact set of $\mathbb{R}_{+}$. For all of these spaces, we use the same convention, as the one used for $\mathbb{R}$ to define matrices of such functions.

\section{Preliminaries and problem formulation}

Consider, for $t>0$, the LTV system

$$
\begin{aligned}
& \dot{x}(t)=A(t) x(t)+B(t) u(t), \\
& y(t)=C(t) x(t)+D(t) u(t),
\end{aligned}
$$

where $x(t) \in \mathbb{R}^{n}, u(t) \in \mathbb{R}^{m}$ and $y(t) \in \mathbb{R}^{q}$ are respectively the state, the input (or the control) and the output of the system at time $t$ where $n, m$ and $q$ are positive integers. These three integers will always refer to the dimensions of the state, input and output. We also consider $A \in L_{\mathrm{loc}}^{\infty}\left(\mathbb{R}_{+}\right)_{n}^{n}, B \in L_{\mathrm{loc}}^{\infty}\left(\mathbb{R}_{+}\right)_{m}^{n}$, $C \in \mathcal{C}^{0}\left(\mathbb{R}_{+}\right)_{n}^{q}$ and $D \in \mathcal{C}^{0}\left(\mathbb{R}_{+}\right)_{m}^{q}$. Unless otherwise stated, these regularities will be assumed all along this paper.

\subsection{Some reminders on differential equations}

Since in this paper, we will investigate the state to output controllability in finite time of system (1), we consider a generic time $t_{f}>0$ and focus on the restrictions of solutions of (1) on the interval $\left[0, t_{f}\right]$. It is well-known, see e.g. [5], Lemma 2], that for $u \in L^{\infty}\left(0, t_{f}\right)^{m}$, equation (1a) coupled with the initial condition $x(0)=\mathrm{x}_{0} \in \mathbb{R}^{n}$ admits a unique solution on $\mathcal{C}^{0}\left(\left[0, t_{f}\right]\right)^{n}$ defined, for every $t \in\left[0, t_{f}\right]$, by

$$
x_{u}\left(\mathrm{x}_{0}, t\right)=R(t, 0) \mathrm{x}_{0}+\int_{0}^{t} R(t, s) B(s) u(s) \mathrm{d} s .
$$

This solution is expressed in terms of the resolvent matrix $R$ associated to the system $\dot{x}(t)=A(t) x(t)$. Definition and properties of this matrix are recalled below.

Definition 1 The resolvent of the system $\dot{x}(t)=A(t) x(t)$ is the map $R:(t, \tau) \in$ $\mathbb{R}_{+}^{2} \mapsto R(t, \tau) \in \mathbb{R}_{n}^{n}$ such that for every $(t, \tau) \in \mathbb{R}_{+}^{2}, M(t)=R(t, \tau)$ is the unique solution of the Cauchy problem $\dot{M}(t)=A(t) M(t)$ with $M(\tau)=I_{n}$.

The following properties of this map can be found for instance in [1, Lecture 18].

Proposition 1 We have $R \in \mathcal{C}^{0}\left(\mathbb{R}_{+}^{2}\right)_{n}^{n}$, and for every $t_{0}, t_{1}, t_{2} \in \mathbb{R}_{+}, R\left(t_{0}, t_{0}\right)=$ $I_{n}$ and $R\left(t_{0}, t_{1}\right) R\left(t_{1}, t_{2}\right)=R\left(t_{0}, t_{2}\right)$. In addition, if $A \in \mathcal{C}^{k}\left(\mathbb{R}_{+}\right)_{n}^{n}$, with $k \in \mathbb{N}$, then $R \in \mathcal{C}^{k+1}\left(\mathbb{R}_{+}^{2}\right)_{n}^{n}$ and for every $t \in \mathbb{R}_{+}$,

$$
\frac{\partial R\left(t, t_{2}\right)}{\partial t}=A(t) R\left(t, t_{2}\right), \quad \frac{\partial R\left(t_{1}, t\right)}{\partial t}=-R\left(t_{1}, t\right) A(t) .
$$




\subsection{Definition and problem statement}

In the framework of state controllability, it is well-known, and can be seen through (2), that even if we consider the set of essentially bounded controls as a set of admissible inputs, the state trajectories are continuous. But, when one wants to control the output of the system (1) with $D \neq 0_{m}^{q}$, one can readily see that allowing jumps on the input induces discontinuities on the output trajectories. This is often not desirable in practice. For this practical purpose, it is more natural to consider the set of continuous functions as the set of admissible controls. Therefore, the following definition will be considered in the sequel.

Definition 2 The system (1) is said to be state to output controllable (SOC) in time $t_{f}$, if for every $\left(\mathrm{x}_{0}, \mathrm{y}_{1}\right) \in \mathbb{R}^{n} \times \mathbb{R}^{q}$, there exists a control $u \in \mathcal{C}^{0}\left(\left[0, t_{f}\right]\right)^{m}$ such that the output solution $y_{u}\left(\mathrm{x}_{0}, t\right)$ of system (1) with $\mathrm{x}_{0} \in \mathbb{R}^{n}$ as initial state data and $u$ as input satisfies at time $t_{f}, y_{u}\left(\mathrm{x}_{0}, t_{f}\right)=\mathrm{y}_{1}$.

This definition gives rise to the following problems.

Problem 1 Determine efficient and practical characterizations of the SOC in finite time of system (1).

Problem 2 For any $\left(\mathrm{x}_{0}, \mathrm{y}_{1}\right) \in \mathbb{R}^{n} \times \mathbb{R}^{q}$, find, when system (1) is SOC in a time $t_{f}>0$, an explicit expression of a continuous control steering $\mathrm{x}_{0}$ to $\mathrm{y}_{1}$ in time $t_{f}$ in the sense of Definition 2.

From $1 \mathrm{~b}$, we observe that in any case, $y_{u}\left(\mathrm{x}_{0}, t_{f}\right) \in \operatorname{Im}\left(C\left(t_{f}\right) \mid D\left(t_{f}\right)\right)$. Hence, a necessary condition for system (1) to be SOC in time $t_{f}$ is $\operatorname{rk}\left(C\left(t_{f}\right) \mid D\left(t_{f}\right)\right)=q$. This condition also tells us that if system (1) is SOC in some time $t_{f}>0$, it is not necessarily SOC in another time $\tilde{t}_{f} \neq t_{f}$.

\section{Main results}

The proofs of the results given in this section are postponed in Section 5. All along this paper, the following notations will be used: for all $t \in\left[0, t_{f}\right]$ and $u \in \mathcal{C}^{0}\left(\left[0, t_{f}\right]\right)^{m}$,

$$
\begin{aligned}
M_{t_{f}}(t) & :=C\left(t_{f}\right) R\left(t_{f}, t\right) B(t) \in \mathbb{R}_{m}^{q}, \\
H_{t_{f}}(t) & :=\int_{t}^{t_{f}} M_{t_{f}}(s) \mathrm{d} s+D\left(t_{f}\right) \in \mathbb{R}_{m}^{q}, \\
E_{t_{f}}(u) & :=\int_{0}^{t_{f}} M_{t_{f}}(t) u(t) \mathrm{d} t+D\left(t_{f}\right) u\left(t_{f}\right) \in \mathbb{R}^{q}, \\
\mathcal{K}_{t_{f}} & :=\int_{0}^{t_{f}} M_{t_{f}}(t) M_{t_{f}}(t)^{\top} \mathrm{d} t+D\left(t_{f}\right) D\left(t_{f}\right)^{\top} \in \mathbb{R}_{q}^{q}, \\
\mathcal{G}_{t_{f}} & :=\int_{0}^{t_{f}} H_{t_{f}}(t) H_{t_{f}}(t)^{\top} \mathrm{d} t \in \mathbb{R}_{q}^{q} .
\end{aligned}
$$

The following theorem links the notion of SOC in time $t_{f}$ to the surjectivity of the Endpoint map $E_{t_{f}}$, and to the positive definiteness of the Gramian matrices $\mathcal{K}_{t_{f}}$ and $\mathcal{G}_{t_{f}}$.

Theorem 2 The following assertions are equivalent.

(i) The system (1) is SOC in time $t_{f}$. 
(ii) The map $E_{t_{f}}$ defined on $\mathcal{C}^{0}\left(\left[0, t_{f}\right]\right)^{m}$ is surjective.

(iii) The symmetric matrix $\mathcal{K}_{t_{f}}$ is positive definite.

(iv) The symmetric matrix $\mathcal{G}_{t_{f}}$ is positive definite.

We will see in Theorem 8 that the Gramian matrix $\mathcal{G}_{t_{f}}$, involved in condition (iv)] will also lead to an expression of a continuous control, while the Gramian matrix $\mathcal{K}_{t_{f}}$ proposed in [6] will only lead to a piecewise continuous control.

The above SOC criteria (iii) and (iv) might be difficult to check in practice. Indeed, to use them, we need to compute the resolvent matrix, and to check the invertibility of a matrix. This is difficult in general, even in low dimensions. For that reason, we propose in Theorem 3 a sufficient condition which does not require the knowledge of the resolvent matrix. Furthermore, this condition becomes necessary under analytical assumption.

Theorem 3 Let $k \in \mathbb{N} \cup\{\infty\}$, and assume that $A \in \mathcal{C}^{k-1}\left(\mathbb{R}_{+}\right)_{n}^{n}$ (or $A \in$ $L_{\text {loc }}^{\infty}\left(\mathbb{R}_{+}\right)_{n}^{n}$ for $\left.k=0\right), B \in \mathcal{C}^{k}\left(\mathbb{R}_{+}\right)_{m}^{n}$. If there exists a time $t_{f}>0$ such that

$$
\operatorname{rk}\left(D\left(t_{f}\right) \mid C\left(t_{f}\right) B_{i}\left(t_{f}\right), i \in \mathbb{N}_{\leqslant k}\right)=q,
$$

where $B_{0}(t)=-B(t)$ and $B_{i+1}(t)=-\dot{B}_{i}(t)+A(t) B_{i}(t)$, then system (1) is $S O C$ in time $t_{f}$. Moreover, if $A$ and $B$ are analytic matrices, then (3), with $k=\infty$, is necessary.

Remark 1 Theorem 3 is also valid if the matrices $A(t)$ and $B(t)$ only have the required regularity at time $t=t_{f}$.

Remark 2 Since the rank of a matrix is invariant under a certain threshold of perturbation (see e.g. [8, Chapter 5]), if (3) holds in time $t_{f}$, then (3) also holds in a neighborhood of $t_{f}$. Indeed, one can see that the matrices $D$ and $C B_{i}$ (for $i \in \mathbb{N}_{\leqslant k}$ ) are all continuous at time $t_{f}$. Furthermore, if $A(t), B(t), C(t)$ and $D(t)$ are analytic matrices with respect to $t$, and (3) holds for some time $t_{f}>0$ and some $k \in \mathbb{N}$, then (3) holds for almost every $t_{f} \in \mathbb{R}_{+}$.

Remark 3 Bearing in mind Cayley-Hamilton theorem, one can readily see that if matrices $A, B, C$ and $D$ are time independent, condition (3), with $k \geqslant n$, is exactly the Kalman extended rank criterion given in [4. Theorem $3.1\left(\operatorname{soc}_{2}\right)$ ], that is $\operatorname{rk}\left(D\left|C A^{0} B\right| \cdots \mid C A^{n-1} B\right)=q$. If $A$ and $B$ are not constant, we can no longer use Cayley-Hamilton in (3) due to the presence of the time derivatives of these matrices.

We insist on the fact that condition (3) is sufficient, but not necessary as it is illustrated on the following example.

Example 1 Consider system (1) with $A=0_{3}^{3}, D=0_{1}^{2}, C=\left(I_{2} \mid 0_{1}^{2}\right)$ and $B(t)=(f(t), g(t), h(t))^{\top}$, where $f, g, h \in \mathcal{C}^{k}\left(\mathbb{R}_{+}\right)$, for some $k \in \mathbb{N} \cup\{\infty\}$, non identically null functions on $\left[0, t_{f}\right]$ such that the support of $f$ and $g$ are respectively included in $\left[0, t_{f} / 2\right]$ and $\left[t_{f} / 2, t_{f}\right]$. We deduce that $\operatorname{Span}\left\{D\left(t_{f}\right) u, u \in\right.$ 
$\left.\mathbb{R}^{m}\right\}+\operatorname{Span}\left\{C\left(t_{f}\right) B_{i}\left(t_{f}\right) u, i \in \mathbb{N}_{\leqslant k}, u \in \mathbb{R}^{m}\right\} \subset\{0\} \times \mathbb{R}$. Therefore, we infer that condition (3) does not hold in time $t_{f}$. But one can see through condition (iii) that $\mathcal{K}_{t_{f}}=\operatorname{diag}\left(\int_{0}^{t_{f}} f(t)^{2} \mathrm{~d} t, \int_{0}^{t_{f}} g(t)^{2} \mathrm{~d} t\right)$ is positive definite, thanks to the assumptions made on $f$ and $g$.

Contrary to Theorem 3 , the following results use the resolvent matrix $R$. Thus, they are only usable in practice if one knows how to calculate it. For the sake of completeness, we recall in the following lemma the expression of the derivatives of $H_{t_{f}}$. The proof of this lemma is not given but can be obtained by induction, together with Proposition 1

Lemma 4 Let $k \in \mathbb{N} \cup\{+\infty\}$. If $A \in \mathcal{C}^{k-1}\left(\mathbb{R}_{+}\right)_{n}^{n}$ (or $A \in L_{\text {loc }}^{\infty}\left(\mathbb{R}_{+}\right)_{n}^{n}$ for $k=0$ ), $B \in \mathcal{C}^{k}\left(\mathbb{R}_{+}\right)_{m}^{n}$ then $H_{t_{f}} \in \mathcal{C}^{k+1}\left(\mathbb{R}_{+}\right)_{m}^{q}$ and $H_{t_{f}}^{(i)}(t)=C\left(t_{f}\right) R\left(t_{f}, t\right) B_{i-1}(t)$ for all $i \in \mathbb{N}_{\leqslant k+1}^{*}$ and $t \in \mathbb{R}_{+}$.

The following proposition extends [2, Proposition 1.19] to the case of the controllability of the output of system (1).

Proposition 5 Assume there exists a time $t_{f}>0$ such that (3) holds with $k=\infty$. Then there exists $\varepsilon>0$ such that for every $t \in\left(t_{f}-\varepsilon, t_{f}+\varepsilon\right) \cap\left(\mathbb{R}_{+} \backslash\left\{t_{f}\right\}\right)$,

$$
\operatorname{rk}\left(H_{t_{f}}^{(i)}(t), i \in \mathbb{N}_{<q}\right)=q,
$$

Moreover, if $A$ and $B$ are analytic then (3) and (4) are equivalent.

Remark 4 Without the analyticity hypothesis, (3) and (4) are not equivalent. One can get convinced by considering system (1) with $m=n=q=1, A=D=$ $0, C=1$ and $B(t)=\left(2 /(t-1)^{3}\right) e^{-1 /(t-1)^{2}}$ for $t \neq 1$ and $B(1)=0$. With this example, one can easily see that taking $t_{f}=1$, (4) holds but (3) does not.

The following theorem generalizes Theorems 3 and Proposition 5 . The proof of this theorem will be sketched at the same time as the one of Theorems 3 and Proposition 5 .

Theorem 6 Let $k \in \mathbb{N} \cup\{\infty\}$. Assume that $A \in \mathcal{C}^{k-1}\left(\mathbb{R}_{+}\right)_{n}^{n}\left(\right.$ or $A \in L_{\text {loc }}^{\infty}\left(\mathbb{R}_{+}\right)_{n}^{n}$ for $k=0), B \in \mathcal{C}^{k}\left(\mathbb{R}_{+}\right)_{m}^{n}$ and let $\ell \in \mathbb{N}_{\leqslant q}^{*}$. The system (1) is SOC in time $t_{f}$ if there exist $t_{1}, \cdots, t_{\ell}$ in $\left[0, t_{f}\right]$, not necessarily distinct, such that

$$
\operatorname{rk}\left(H_{t_{f}}^{(i)}\left(t_{j}\right), i \in \mathbb{N}_{\leqslant k+1}, j \in \mathbb{N}_{\leqslant \ell}^{*}\right)=q,
$$

Moreover, if (5) holds with $k=\infty$ and $\ell=1$, then there exists $\varepsilon>0$ such that for every $t \in\left(t_{1}-\varepsilon, t_{1}+\varepsilon\right) \cap\left(\left[0, t_{f}\right] \backslash\left\{t_{1}\right\}\right)$, we have $\operatorname{rk}\left(H_{t_{f}}^{(i)}(t), i \in \mathbb{N}_{<q}\right)=q$.

Let us compare Theorem 6 with [ 3 , Theorem 4.9]. In [3, Theorem 4.9], it is required that the regularity of the matrices $A$ and $B$ are respectively $n-2$ and $n-1$, and the rank condition shall be satisfied on a nonempty interval. In Theorem 6 we do not really have regularity constraints, and the rank condition 
is pointwise. Needless to say that if the necessary condition of [3, Theorem 4.9] is fulfilled, then the necessary condition of Theorem 6 is also fulfilled. In addition, all the discussions that we had in the analytic case can be applied to Theorem 6 .

As title of example, let us apply Theorem 6 to Example 1 with $k=0$, i.e., the functions $f, g$ and $h$ are just continuous. In that case, $H_{t_{f}} \in \mathcal{C}^{1}\left(\mathbb{R}_{+}\right)_{2}$, $H_{t_{f}}(t)=\left(\int_{t}^{t_{f}} f(s) \mathrm{d} s, \int_{t}^{t_{f}} g(s) \mathrm{d} s\right)^{\top}$ and $H_{t_{f}}^{(1)}(t)=-(f(t), g(t))^{\top}$ for all $t \in$ $\left[0, t_{f}\right]$. By assumption, there exists $\left(t_{1}, t_{2}\right) \in\left[0, t_{f} / 2\right] \times\left[t_{f} / 2, t_{f}\right]$ such that $f\left(t_{1}\right) \neq 0, f\left(t_{2}\right)=0, g\left(t_{1}\right)=0$ and $g\left(t_{2}\right) \neq 0$. From these computations, we have $\operatorname{rk}\left(H_{t_{f}}^{(1)}\left(t_{1}\right), H_{t_{f}}^{(1)}\left(t_{2}\right)\right)=q=2$. Hence, Theorem 6 ensures the SOC of the system in time $t_{f}$.

In the case we do not have enough regularity to apply Theorems 3 or 6 . the following theorem can be considered. This theorem, while being simple, is relevant since it gives necessary and sufficient condition for SOC without requiring supplementary regularities on the matrices $A$ and $B$ other than being essentially bounded. Recall that in that case, $H_{t_{f}} \in \mathcal{C}^{0}\left(\mathbb{R}_{+}\right)_{m}^{q}$.

Theorem 7 The system (1) is SOC in time $t_{f}$ if and only if there exists $t_{1}, \cdots, t_{q} \in\left[0, t_{f}\right]$, not necessarily distinct, such that

$$
\operatorname{rk}\left(H_{t_{f}}\left(t_{1}\right), \cdots, H_{t_{f}}\left(t_{q}\right)\right)=q .
$$

Moreover, if $A$ and $B$ are analytic, $\left(t_{1}, \cdots, t_{q}\right)$ can be found in any nonempty open set contained in $\left[0, t_{f}\right]^{q}$.

Since the rank of a matrix is invariant under a certain threshold of perturbation, if there exists $\left(t_{1}, \cdots, t_{q}\right) \in\left[0, t_{f}\right]^{q}$ such that (6) holds, then there exists a neighborhood $\mathcal{N}$ of $\left(t_{1}, \cdots, t_{q}\right)$ in $\left[0, t_{f}\right]^{q}$ such that for every $\left(\tilde{t}_{1}, \cdots, \tilde{t}_{q}\right) \in \mathcal{N}$, $\operatorname{rk}\left(H_{t_{f}}\left(\tilde{t}_{1}\right), \ldots, H_{t_{f}}\left(\tilde{t}_{q}\right)\right)=q$. From the continuity of $H_{t_{f}}$ with respect to $t_{f}$ if (6) holds, there exists $\varepsilon>0$ such that for every $\tilde{t}_{f} \in\left(t_{f}-\varepsilon, t_{f}+\varepsilon\right) \cap \mathbb{R}_{+}$the system is SOC in time $\tilde{t}_{f}$.

To illustrate Theorem 7, let us consider again Example 1, with $f$ and $g$ uniformly bounded. Due to the support assumptions made on $f$ and $g$, there exist $\left(t_{1}, t_{2}\right) \in$ $\left[0, t_{f} / 2\right] \times\left[t_{f} / 2, t_{f}\right]$ such that $\int_{t_{1}}^{t_{f}} f(s) \mathrm{d} s \neq 0, \int_{t_{2}}^{t_{f}} f(s) \mathrm{d} s=0$ and $\int_{t_{2}}^{t_{f}} g(s) \mathrm{d} s \neq 0$. That is to say that $\operatorname{rk}\left(H_{t_{f}}\left(t_{1}\right), H_{t_{f}}\left(t_{2}\right)\right)=q=2$, and hence, according to Theorem 7 , this system is controllable.

We end this section by giving Theorem 8 which solves Problem 2

Theorem 8 Assume that the system (1) is SOC in time $t_{f}$. For every $\left(\mathrm{x}_{0}, \mathrm{y}_{1}\right) \in$ $\mathbb{R}^{n} \times \mathbb{R}^{q}$ and every $\mathrm{u}_{0} \in \mathbb{R}^{m}$, the control, given, for every $t \in\left[0, t_{f}\right]$, by

$$
u(t)=\mathrm{u}_{0}+\int_{0}^{t} H_{t_{f}}(\tau)^{\top}\left(\mathcal{G}_{t_{f}}\right)^{-1}\left(\mathrm{y}_{1}-y_{\mathrm{u}_{0}}\left(\mathrm{x}_{0}, t_{f}\right)\right) \mathrm{d} \tau,
$$

steers $\mathrm{x}_{0}$ to $\mathrm{y}_{1}$ in time $t_{f}$, where we have set $y_{\mathrm{u}_{0}}\left(\mathrm{x}_{0}, t_{f}\right)=C\left(t_{f}\right) R\left(t_{f}, 0\right) \mathrm{x}_{0}+$ $H_{t_{f}}(0) \mathrm{u}_{0}$.

Furthermore, this control is the unique minimizer of

$$
\begin{aligned}
& \min \frac{1}{2} \int_{0}^{t_{f}}|\dot{w}(t)|^{2} \mathrm{~d} t \\
& w \in H^{1}\left(0, t_{f}\right)^{m}, w(0)=\mathrm{u}_{0}, \mathrm{y}_{1}=y_{w}\left(\mathrm{x}_{0}, t_{f}\right) .
\end{aligned}
$$


Remark 5 The expression of the control given in (7) is based on the inverse of the Gramian matrix $\mathcal{G}_{t_{f}}$. As already mentioned, computing this matrix and checking its invertibility might be hard in practice. From a numerical point of view, it is more interesting to consider the minimization problem (8) than trying to compute directly the expression (7).

When system (1) is SOC in time $t_{f}>0$, even if the matrix $\mathcal{K}_{t_{f}}$ does not lead to a continuous control as mentioned earlier, we observe from [ $[\underline{6}$ that it leads to a piecewise continuous one. More precisely, the control given by

$$
\left\{\begin{array}{l}
u(t)=M_{t_{f}}(t)^{\top}\left(\mathcal{K}_{t_{f}}\right)^{-1} \delta_{\mathrm{y}}, \quad \text { for } t \in\left[0, t_{f}\right), \\
u\left(t_{f}\right)=D\left(t_{f}\right)^{\top}\left(\mathcal{K}_{t_{f}}\right)^{-1} \delta_{\mathrm{y}},
\end{array}\right.
$$

with $\delta_{\mathrm{y}}=\mathrm{y}_{1}-C\left(t_{f}\right) R\left(t_{f}, 0\right) \mathrm{x}_{0}$, steers $\mathrm{x}_{0}$ to $\mathrm{y}_{1}$ in time $t_{f}$. Furthermore, this control is the unique minimizer of

$$
\begin{aligned}
\min & \frac{1}{2} \int_{0}^{t_{f}}|u(t)|^{2} \mathrm{~d} t+\frac{1}{2}|\xi|^{2} \\
& u \in L^{2}\left(0, t_{f}\right)^{m}, \quad \xi \in \mathbb{R}^{m}, \\
& \mathrm{y}_{1}-C\left(t_{f}\right) R\left(t_{f}, 0\right) \mathrm{x}_{0}=\int_{0}^{t_{f}} M_{t_{f}}(t) u(t) \mathrm{d} t+D\left(t_{f}\right) \xi,
\end{aligned}
$$

where $\xi$ stands for the final control value $u\left(t_{f}\right)$. One can see from the above expression of $u$ that, this control is discontinuous unless $C\left(t_{f}\right) B\left(t_{f}\right)=D\left(t_{f}\right)$.

\section{Illustration}

To illustrate our results, we consider the system (1) with $n=3, m=1, q=2$, and matrices $A, B, C$ and $D$ given for $t \geqslant 0$ by

$$
\begin{array}{ll}
A(t)=\left(\begin{array}{rrc}
0 & -1 & 0 \\
1 & 0 & 0 \\
0 & 0 & t
\end{array}\right), & B(t)=\left(\begin{array}{c}
\cos (t) \\
\sin (t) \\
0
\end{array}\right), \\
C(t)=\left(\begin{array}{rrc}
0 & 1 & t^{2} \\
t & 0 & \cos (t)
\end{array}\right), & D(t)=\left(\begin{array}{c}
0 \\
\sin (t)
\end{array}\right) .
\end{array}
$$

For any time $t_{f}>0$, one can check that the state controllability Gramian in time $t_{f}$, given in [2, Theorem 1.11] applied to system (1) with (10) is not invertible. It follows that this system is not state controllable in time $t_{f}$.

- Criteria (iii) and (iv). Let us check if this system is SOC in some time $t_{f}>0$. For any time $t_{f}>0$, we get after some computations $\operatorname{det}\left(\mathcal{K}_{t_{f}}\right)=$ $t_{f} \sin \left(t_{f}\right)^{4}$ and $\operatorname{det}\left(\mathcal{G}_{t_{f}}\right)=t_{f}^{4} \sin \left(t_{f}\right)^{4} / 12$, where $\mathcal{K}_{t_{f}}$ and $\mathcal{G}_{t_{f}}$ are the matrices defined respectively in (iii) and (iv)] It follows from Theorem 2 that system (1) with matrices given in (10) is SOC in time $t_{f}>0$ if and only if $t_{f} \notin \pi \mathbb{N}$.

- Theorem 3. Since the matrices defined in (10) are analytic, condition (3) turns to be necessary and sufficient. From (iv) we have $H_{t_{f}}(t)=\left(\sin \left(t_{f}\right)\left(t_{f}-\right.\right.$ $\left.t), \sin \left(t_{f}\right)+t_{f} \cos \left(t_{f}\right)\left(t_{f}-t\right)\right)^{\top}$. We then have $H_{t_{f}}^{(0)}(t)=H_{t_{f}}(t), H_{t_{f}}^{(1)}(t)=$ 
$-\left(\sin \left(t_{f}\right), t_{f} \cos \left(t_{f}\right)\right)^{\top}$ and $H_{t_{f}}^{(i)}(t)=0$ for all $i \geqslant 2$. It follows from the previous computations that system (1) with (10) is SOC in time $t_{f}$ if and only if $t_{f} \notin \pi \mathbb{N}$. - Proposition 5. For every $t>0$, we have $\operatorname{det}\left(H_{t_{f}}^{(i)}(t), i \in \mathbb{N}_{<2}\right)=\sin \left(t_{f}\right)^{2}$. It follows that system (1) with (10) is SOC in time $t_{f}$ if and only if criterion (3) is fulfilled. Here again, we have used the analyticity of the matrices defined in (10) to get the necessary condition.

- Theorem 7 For every $t_{1}, t_{2} \in\left[0, t_{f}\right]$, we have $\operatorname{det}\left(H_{t_{f}}\left(t_{1}\right), H_{t_{f}}\left(t_{2}\right)\right)=\left(t_{2}-\right.$ $\left.t_{1}\right) \sin \left(t_{f}\right)$. We deduce that $\left(t_{1}, t_{2}\right) \mapsto \operatorname{det}\left(H_{t_{f}}\left(t_{1}\right), H_{t_{f}}\left(t_{2}\right)\right)$ is not identically null if and only if $t_{f} \notin \pi \mathbb{N}$.

- Control computation. To illustrate Theorem 8, we take $t_{f}=\pi / 2$. From the above, the system considered is SOC in this time. Now we would like to build a continuous control $u$, steering $\mathrm{x}_{0}=\left(\begin{array}{lll}1 & 0 & 1\end{array}\right)^{\top}$ to $\mathrm{y}_{1}=\left(\begin{array}{ll}1 & 2\end{array}\right)^{\top}$ in time $t_{f}$. For this purpose, we decide to construct $u$ such that $u(0)=1$. Applying formula (7), we get for every $t \in[0, \pi / 2]$

$$
u(t)=\frac{12}{\pi^{2}}\left(3+\pi e^{\pi^{2} / 8}\right) t^{2}-\frac{2}{\pi}\left(8+3 \pi e^{\pi^{2} / 8}\right) t+1 .
$$

One can also see that the piecewise control built in $(9)$ is given by $u(t)=$ $-\frac{\pi}{2} e^{\pi^{2} / 8}$ for $t \in[0, \pi / 2)$ and $u\left(\frac{\pi}{2}\right)=2$. Solving (1) with these two controls, and $\mathrm{x}_{0}$ as initial data, we get the time trajectories depicted on Figure 1.

\section{Proofs of the results}

\subsection{Proof of Theorem 2}

We do not give the proof of condition (iii) since it can be found in [6, Theorem 2]. We only prove the equivalence between (i) (ii) and (iv)

- Equivalence between (i) and[(ii). First note that if system (1) is SOC in time $t_{f}$ then the origin can be steered to any point of $\mathbb{R}^{q}$ in time $t_{f}$. This implies that (ii) holds. Reciprocally, if $E_{t_{f}}$ is surjective, for every $\left(\mathrm{x}_{0}, \mathrm{y}_{1}\right) \in \mathbb{R}^{n} \times \mathbb{R}^{q}$, there exists a control $u^{*} \in \mathcal{C}^{0}\left(\left[0, t_{f}\right]\right)^{m}$ such that $E_{t_{f}}\left(u^{*}\right)=\mathrm{y}_{1}-C\left(t_{f}\right) R\left(t_{f}, 0\right) \mathrm{x}_{0}$, i.e., $\mathrm{x}_{0}$ can be steered to $\mathrm{y}_{1}$ in time $t_{f}$.

- Equivalence between (ii) and (iv), Let us first assume that there exists a non-zero vector $\eta \in \mathbb{R}^{q}$ such that

$$
\eta^{\top} \mathcal{G}_{t_{f}} \eta=0
$$

Since $H_{t_{f}} \in \mathcal{C}^{0}\left(\mathbb{R}_{+}\right)_{m}^{q}$, we deduce that $\eta^{\top} H_{t_{f}}(t)=0_{m}^{1}$ for all $t \in\left[0, t_{f}\right]$, i.e.

$$
\eta^{\top} \int_{t}^{t_{f}} M_{t_{f}}(s) \mathrm{d} s+\eta^{\top} D\left(t_{f}\right)=0_{m}^{1}, \quad \forall t \in\left[0, t_{f}\right] .
$$

Taking $t=t_{f}$ in $(12)$ and differentiating the same equation with respect to $t$, one deduce that $\eta^{\top} D\left(t_{f}\right)=0_{m}^{1}$ and $\eta^{\top} M_{t_{f}}(t)=0_{m}^{1}$ for almost every $t \in\left[0, t_{f}\right]$. 

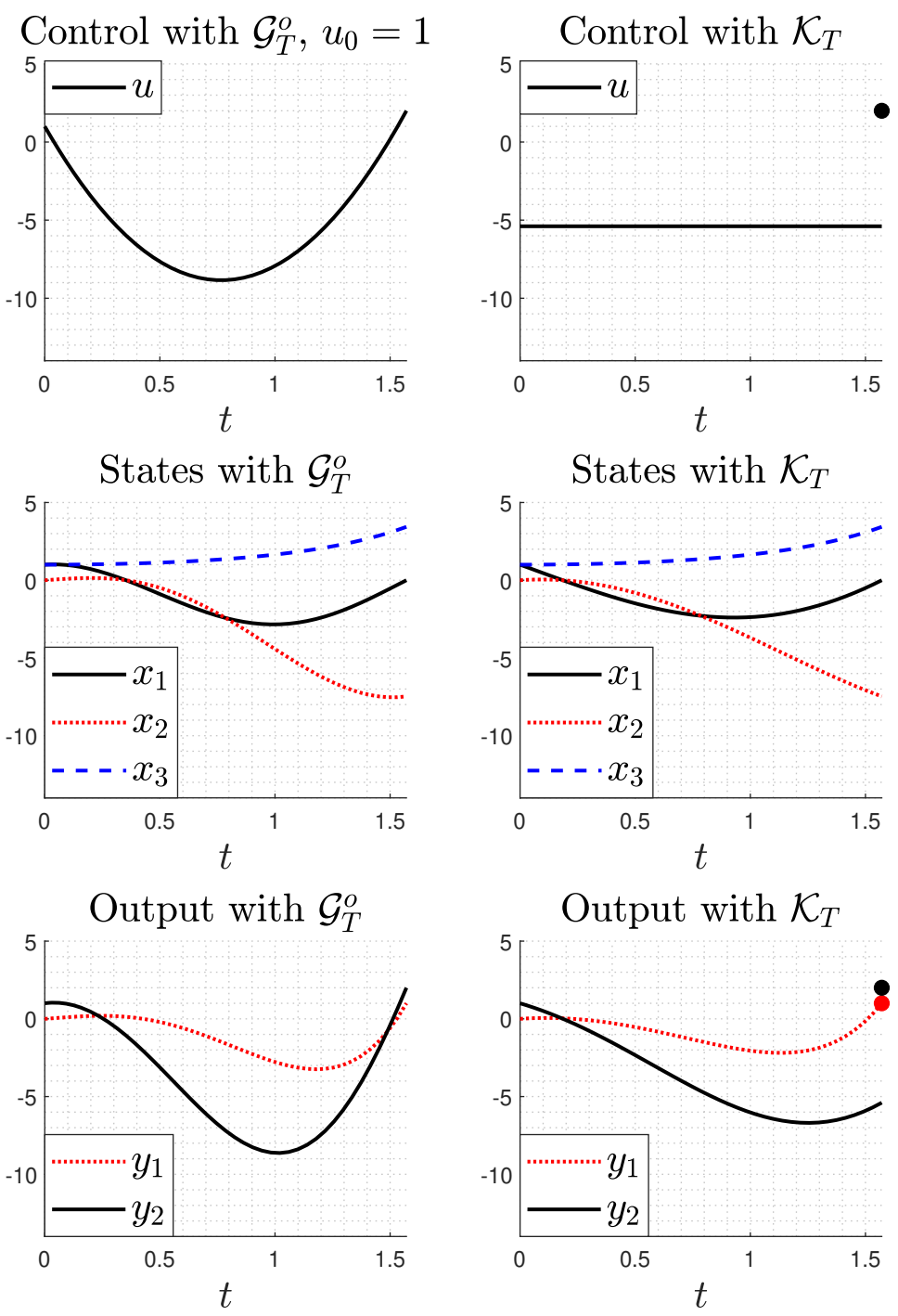

Figure 1: On the left, we have the continuous control (Theorem 8), state and output trajectories built by means of the matrix $\mathcal{G}_{t_{f}}$ taking the output of system (10) to $\mathrm{y}_{1}=\left(\begin{array}{lll}1 & 2\end{array}\right)^{\top}$ in time $t_{f}=\pi / 2$ with $\mathrm{x}_{0}=\left(\begin{array}{lll}1 & 0 & 1\end{array}\right)^{\top}$ as initial state data. On the right-hand side, we have the time trajectories obtained using the control built with $\mathcal{K}_{t_{f}}$. One can see that the output trajectories with our proposed Gramian $\mathcal{G}_{t_{f}}$ are continuous and those built by means of $\mathcal{K}_{t_{f}}$ are not.

It follows that the linear map $E_{t_{f}}$ is not surjective.

Conversely, assume there exists $\eta \in \mathbb{R}^{q} \backslash\left\{0^{q}\right\}$ such that $\eta^{\top} E_{t_{f}}(u)=0$ for every $u \in \mathcal{C}^{0}\left(\left[0, t_{f}\right]\right)^{m}$. It follows that $\eta^{\top} \int_{0}^{t_{f}} M_{t_{f}}(t) u(t) \mathrm{d} t+\eta^{\top} D\left(t_{f}\right) u\left(t_{f}\right)=0$, for 
every $u \in \mathcal{C}^{1}\left(\left[0, t_{f}\right]\right)^{m}$, and therefore, for every $v \in \mathcal{C}^{0}\left(\left[0, t_{f}\right]\right)^{m}$,

$$
\eta^{\top} \int_{0}^{t_{f}} M_{t_{f}}(t)\left[\int_{0}^{t} v(s) \mathrm{d} s\right] \mathrm{d} t+\eta^{\top} D\left(t_{f}\right) \int_{0}^{t_{f}} v(t) \mathrm{d} t=0
$$

Noticing that by an integration by parts we have

$$
\int_{0}^{t_{f}} M_{t_{f}}(t)\left[\int_{0}^{t} v(s) \mathrm{d} s\right] \mathrm{d} t=\int_{0}^{t_{f}} \int_{t}^{t_{f}} M_{t_{f}}(s) v(t) \mathrm{d} s \mathrm{~d} t
$$

we deduce from $(13)$ that

$$
\eta^{\top} \int_{0}^{t_{f}} H_{t_{f}}(t) v(t) \mathrm{d} t=0, \quad \forall v \in \mathcal{C}^{0}\left(\left[0, t_{f}\right]\right)^{m} .
$$

Setting $v(t)=H_{t_{f}}(t)^{\top} \eta$ for all $t \in\left[0, t_{f}\right], v \in \mathcal{C}^{0}\left(\left[0, t_{f}\right]\right)^{m}$ and it follows from (15) that 111 holds for some non-zero vector $\eta$, that is to say that $\mathcal{G}_{t_{f}}$ is not positive definite.

Remark 6 It can be deduced from the above proof that denoting by $\mathcal{R}_{t_{f}}$ the set of all output points that can be reached from $0^{n}$ in time $t_{f}$, we have $\mathcal{R}_{t_{f}}=$ $\left\{E_{t_{f}}(u), u \in \mathcal{C}^{0}\left(\left[0, t_{f}\right]\right)^{m}\right\}=\left\{\int_{0}^{t_{f}} H_{t_{f}}(t) u(t) \mathrm{d} t, u \in \mathcal{C}^{k}\left(\left[0, t_{f}\right]\right)^{m}\right\}$ for every $k \in \mathbb{N}$. This observation shows that [4, Lemma 4.1] remains true in the case of LTV systems.

\subsection{Proof of Theorem 3}

Let us prove Theorem 3 by contraposition. To this end, we assume that system (1) is not SOC in time $t_{f}$. Then, according to Theorem 2, there exists a non-zero vector $\eta \in \mathbb{R}^{q}$ such that $\eta^{\top} \mathcal{G}_{t_{f}} \eta=0$. Using the continuity of $H_{t_{f}}$, we infer that

$$
\eta^{\top} H_{t_{f}}(t)=0_{m}^{1}, \quad \forall t \in\left[0, t_{f}\right] .
$$

Taking $t=t_{f}$ in (16), we get $\eta^{\top} D\left(t_{f}\right)=0_{m}^{1}$. Using Lemma 4, we deduce by differentiating (16) with respect to $t$ and evaluating the resulting equations at time $t_{f}$ that for every $i \in \mathbb{N}_{\leqslant k}, \eta^{\top} C\left(t_{f}\right) B_{i}\left(t_{f}\right)=0_{m}^{1}$. From the above, we deduce that (3) does not hold. Note that in (16), we decided to evaluate this equality and its derivatives at time $t_{f}$ in order to eliminate the resolvent. But one can also evaluate this relation and its derivatives at any other time taken in $\left[0, t_{f}\right]$. This leads to the sufficient condition given in Theorem 6 .

Observe that if in addition, matrices $A$ and $B$ are analytic, then the map $t \mapsto$ $\eta^{\top} H_{t_{f}}(t)$ is analytic in time. It follows that if its derivatives of all order vanish at some time, then that map vanishes identically on $\mathbb{R}_{+}$. This implies that $\mathcal{G}_{t_{f}} \eta=0^{q}$ and therefore that $\mathcal{G}_{t_{f}}$ is not positive definite. 


\subsection{Proof of Proposition 5}

The proof of this proposition is divided into two steps. The first step deals with the case $m=1$. In the second step, we show how the case $m>1$ can be reduced to the case $m=1$. This proof is inspired by the proof of [2, Proposition 1.19]. Note that when $A$ and $B$ are analytic, the reverse implication is deduced from Theorem 3 .

- Case $m=1$. Note that since $A \in \mathcal{C}^{\infty}\left(\mathbb{R}_{+}\right)_{n}^{n}$ and $B \in \mathcal{C}^{\infty}\left(\mathbb{R}_{+}\right)^{n}$, then $H_{t_{f}} \in \mathcal{C}^{\infty}\left(\mathbb{R}_{+}\right)^{q}$. Since (3) holds for $t_{f}$ thanks to the assumption in Proposition 5. we have from (3) and Lemma 4. $\operatorname{rk}\left(H_{t_{f}}^{(i)}\left(t_{f}\right), i \in \mathbb{N}\right)=q$. Applying [2, Lemma 1.22], and the discussion following this lemma, to the function $H_{t_{f}}$, we deduce that there exist $q$ integers $d_{1}<\cdots<d_{q}, q$ functions $a_{i} \in \mathcal{C}^{\infty}\left(\mathbb{R}_{+}\right)$and $q$ vectors $v_{i} \in \mathbb{R}^{q}$ such that

$$
\begin{gathered}
\operatorname{rk}\left(v_{1}, \cdots, v_{q}\right)=q, \quad a_{i}\left(t_{f}\right) \neq 0, \forall i \in \mathbb{N}_{\leqslant q}^{*}, \\
H_{t_{f}}(t)=\sum_{i=1}^{q} a_{i}(t)\left(t-t_{f}\right)^{d_{i}} v_{i}, \quad \forall t \geqslant 0 .
\end{gathered}
$$

Hence, when $t$ goes to $t_{f}$, we have

$$
\operatorname{det}\left(H_{t_{f}}^{(i)}(t), i \in \mathbb{N}_{<q}\right)=K\left(t-t_{f}\right)^{\delta}+O\left(\left(t-t_{f}\right)^{1+\delta}\right)
$$

with $\delta=-q(q-1) / 2+\sum_{i=1}^{q} d_{i}$ and

$$
K=\left(\prod_{1 \leqslant i<j \leqslant q}\left(d_{j}-d_{i}\right)\right)\left(\prod_{i=1}^{q} a_{i}\left(t_{f}\right)\right) \operatorname{det}\left(v_{1}, \cdots, v_{q}\right) .
$$

From 17a and the fact that $d_{i} \neq d_{j}$ for $i \neq j$, we deduce that $K \neq 0$. It follows from (18) that there exists $\varepsilon>0$ such that for every $t \in\left(t_{f}-\varepsilon, t_{f}+\varepsilon\right) \cap$ $\left(\mathbb{R}_{+} \backslash\left\{t_{f}\right\}\right)$, $\operatorname{det}\left(H_{t_{f}}^{(i)}(t), i \in \mathbb{N}_{<q}\right) \neq 0$. This ends the case $m=1$.

- Case $m>1$. Set $H_{t_{f}}(t)=\left(h_{1}(t), \cdots, h_{m}(t)\right)$. From the hypothesis of Proposition 5 and from Lemma 4 , we have $\mathbb{R}^{q}=\operatorname{Span}\left\{h_{j}^{(i)}\left(t_{f}\right), j \in \mathbb{N}_{\leqslant m}^{*}, i \in \mathbb{N}\right\}$. Hence, there exist $p \in \mathbb{N}^{*}$ such that $\mathbb{R}^{q}=\operatorname{Span}\left\{h_{j}^{(i)}\left(t_{f}\right), j \in \mathbb{N}_{\leqslant m}^{*}, i \in\right.$ $\left.\mathbb{N}_{<p}\right\}$. We then set, for $t \geqslant 0, \tilde{H}(t):=\sum_{j=1}^{m}\left(t-t_{f}\right)^{(j-1) p} h_{j}(t)$ and obtain $\operatorname{rk}\left(\tilde{H}^{(i)}\left(t_{f}\right), i \in \mathbb{N}\right)=q$. Since $h_{j} \in \mathcal{C}^{\infty}\left(\mathbb{R}_{+}\right)^{q}$, we have $\tilde{H} \in \mathcal{C}^{\infty}\left(\mathbb{R}_{+}\right)^{q}$. We therefore use the result of the case $m=1$ to get the existence of $\varepsilon>0$ such that for every $t \in\left(t_{f}-\varepsilon, t_{f}+\varepsilon\right) \cap\left(\mathbb{R}_{+} \backslash\left\{t_{f}\right\}\right)$, rk $\left(\tilde{H}^{(i)}(t), i \in \mathbb{N}_{<q}\right)=q$. Since $\operatorname{Span}\left\{\tilde{H}^{(i)}(t), i \in \mathbb{N}_{<q}\right\} \subset \operatorname{Span}\left\{h_{j}^{(i)}(t), j \in \mathbb{N}_{\leqslant m}^{*}, i \in \mathbb{N}_{<q}\right\}$, we infer that for every $t \in\left(t_{f}-\varepsilon, t_{f}+\varepsilon\right) \cap\left(\mathbb{R}_{+} \backslash\left\{t_{f}\right\}\right), \operatorname{rk}\left(H_{t_{f}}^{(i)}(t), i \in \mathbb{N}_{<q}\right)=q$.

\subsection{Proof of Theorem 7}

It can be seen through $(16)$ that if system (1) is not SOC in time $t_{f}$, condition (6) does not hold for every $t_{1}, \cdots, t_{q}$ in $\left[0, t_{f}\right]$. Assume by contraposition that for 
every $t_{1}, \cdots, t_{q} \in\left[0, t_{f}\right], \operatorname{rk}\left(H_{t_{f}}\left(t_{1}\right), \cdots, H_{t_{f}}\left(t_{q}\right)\right)<q$. Choose $\tau_{1}, \cdots, \tau_{q} \in$ $\left[0, t_{f}\right]$ that realize $\max _{t_{1}, \cdots, t_{q} \in\left[0, t_{f}\right]} \operatorname{rk}\left(H_{t_{f}}\left(t_{1}\right), \cdots, H_{t_{f}}\left(t_{q}\right)\right)$. It follows that for every $u_{\star} \in \mathbb{R}^{m}$ and every $t \in\left[0, t_{f}\right]$,

$$
H_{t_{f}}(t) u_{\star} \in \operatorname{Span}\left(H_{t_{f}}\left(\tau_{i}\right) u, i \in \mathbb{N}_{\leqslant q}^{*}, u \in \mathbb{R}^{m}\right) \subsetneq \mathbb{R}^{q} .
$$

Hence, we deduce that there exists a vector $\eta \in \mathbb{R}^{q} \backslash\left\{0^{q}\right\}$ such that $\eta^{\top} H_{t_{f}}(t)=$ $0_{m}^{1}$ for every $t \in\left[0, t_{f}\right]$. It follows that $\eta$ is in the kernel of $\mathcal{G}_{t_{f}}$, and therefore that system (1) is not SOC in time $t_{f}$. Now, assume that matrices $A$ and $B$ are analytic, and that the system (1) is SOC in time $t_{f}$. From what precedes, there exist $t_{1}, \cdots, t_{q} \in\left[0, t_{f}\right]$ such that $\operatorname{rk}\left(H_{t_{f}}\left(t_{1}\right), \cdots, H_{t_{f}}\left(t_{q}\right)\right)=q$. This implies the existence of $\tau_{1}, \cdots, \tau_{q} \in\left\{t_{1}, \cdots, t_{q}\right\}$ and $u_{1}, \cdots, u_{q} \in \mathbb{R}^{m}$ such that $\operatorname{det}\left(H_{t_{f}}\left(\tau_{1}\right) u_{1}, \cdots, H_{t_{f}}\left(\tau_{q}\right) u_{q}\right) \neq 0$. Since $A$ and $B$ are analytic, the application $\left(s_{1}, \cdots, s_{q}\right) \in\left[0, t_{f}\right]^{q} \mapsto \operatorname{det}\left(H_{t_{f}}\left(s_{1}\right) u_{1}, \cdots, H_{t_{f}}\left(s_{q}\right) u_{q}\right) \in \mathbb{R}$ is also analytic and non identically null. Hence, its zeros set is of null measure. This implies that for every nonempty open set $\mathcal{O}$, in $\left[0, t_{f}\right]^{q}$, there exist $\left(\bar{t}_{1}, \bar{t}_{2}, \cdots, \bar{t}_{q}\right) \in \mathcal{O}$ such that $\operatorname{det}\left(H_{t_{f}}\left(\bar{t}_{1}\right) u_{1}, \cdots, H_{t_{f}}\left(\bar{t}_{q}\right) u_{q}\right) \neq 0$.

\subsection{Proof of Theorem 8}

Let $\mathrm{x}_{0} \in \mathbb{R}^{n}, \mathrm{y}_{1} \in \mathbb{R}^{q}$ and $\mathrm{u}_{0} \in \mathbb{R}^{m}$. Since the system (1) is SOC in time $t_{f}$, there exists a control $u \in \mathcal{C}^{0}\left(\left[0, t_{f}\right]\right)^{m}$ steering $\mathrm{x}_{0}$ to $\mathrm{y}_{1}$ in time $t_{f}$. There even exists an infinite number of controls doing the same transfer since, by the rank Theorem, the kernel of the linear map $E_{t_{f}}$ defined in Theorem 2 is of infinite dimension. We can even build a continuous control such that $u(0)=\mathrm{u}_{0}$ as it will be done in the sequel of this proof. To prove Theorem 8 , we will look for the control solution of the minimization problem (8). We prove this theorem by analysis and synthesis. First suppose that there exists a function $w \in H^{1}\left(0, t_{f}\right)^{m}$ such that $w(0)=\mathrm{u}_{0}$ that steers $\mathrm{x}_{0}$ to $\mathrm{y}_{1}$ in time $t_{f}$. Since $w \in H^{1}\left(0, t_{f}\right)^{m}$ and $w(0)=\mathrm{u}_{0}$, there exists $v \in L^{2}\left(0, t_{f}\right)^{m}$ such that $w(t)=\mathrm{u}_{0}+\int_{0}^{t} v(\tau) \mathrm{d} \tau$. Replacing $w$ by this expression in $y_{w}\left(\mathrm{x}_{0}, t_{f}\right)$ and using (14), we deduce that $y_{w}\left(\mathrm{x}_{0}, t_{f}\right)=y_{\mathrm{u}_{0}}\left(\mathrm{x}_{0}, t_{f}\right)+\Phi(v)$, with $\Phi(v)=\int_{0}^{t_{f}} H_{t_{f}}(t) v(t) \mathrm{d} t$.

The minimization problem (8) can then be rewritten as

$$
\begin{aligned}
& \min \frac{1}{2} \int_{0}^{t_{f}}|v(t)|^{2} \mathrm{~d} t \\
& \quad \mid v \in L^{2}\left(0, t_{f}\right)^{m}, \mathrm{y}_{1}-y_{\mathrm{u}_{0}}\left(\mathrm{x}_{0}, t_{f}\right)=\Phi(v) .
\end{aligned}
$$

Note that problem $(19)$ is the minimization of a strictly convex functional under non-empty affine constraints. Hence, it admits a unique solution in $L^{2}\left(0, t_{f}\right)^{m}$. Taking $\psi$ as the Lagrange multiplier associated to the equality constraint, the Lagrangian of this optimal control problem is given by

$$
\mathcal{L}(v, \psi)=\frac{1}{2} \int_{0}^{t_{f}}|v(t)|^{2} \mathrm{~d} t+\left\langle\psi, \mathrm{y}_{1}-y_{\mathrm{u}_{0}}\left(\mathrm{x}_{0}, t_{f}\right)-\Phi(v)\right\rangle .
$$

Deriving the first order optimality conditions, we get $v(t)=H_{t_{f}}(t)^{\top} \psi$, where $\psi$ is solution of $\mathcal{G}_{t_{f}} \psi=\mathrm{y}_{1}-y_{\mathrm{u}_{0}}\left(t_{f}, \mathrm{x}_{0}\right)$. Since $\mathcal{G}_{t_{f}}$ is invertible, thanks to the 
SOC in time $t_{f}$ of system (1), we deduce that $\psi=\left(\mathcal{G}_{t_{f}}\right)^{-1}\left(\mathrm{y}_{1}-y_{\mathrm{u}_{0}}\left(\mathrm{x}_{0}, t_{f}\right)\right)$. Of the foregoing, we have $v(t)=H_{t_{f}}(t)^{\top}\left(\mathcal{G}_{t_{f}}\right)^{-1}\left(\mathrm{y}_{1}-y_{\mathrm{u}_{0}}\left(\mathrm{x}_{0}, t_{f}\right)\right)$, and $w(t)=$ $\mathrm{u}_{0}+\int_{0}^{t} H_{t_{f}}(\tau)^{\top}\left(\mathcal{G}_{t_{f}}\right)^{-1}\left(\mathrm{y}_{1}-y_{\mathrm{u}_{0}}\left(\mathrm{x}_{0}, t_{f}\right)\right) \mathrm{d} \tau$. Finally, setting $u=w$, we have $u \in \mathcal{C}^{0}\left(\left[0, t_{f}\right]\right)^{m}$ with $u(0)=\mathrm{u}_{0}$ and by construction, this control steers $\mathrm{x}_{0}$ to $\mathrm{y}_{1}$ in time $t_{f}$.

\section{Conclusion}

In this paper, we provided criteria for state to output controllability of linear time varying control systems. When the system is state to output controllable, we gave an explicit expression of a continuous control realizing the desired transfer, this even if there is a direct transmission between the input and the output. In this paper, we have assumed that the initial state is known. However, combining the arguments used in this paper and the strategy used in [4, Section 6], one can also derive similar conditions for the output to output notions introduced in [4.

\section{References}

[1] R. P. Agarwal and D. O'Regan. An introduction to ordinary differential equations. Universitext. Springer, New York, 2008.

[2] J.-M. Coron. Control and nonlinearity, volume 136 of Mathematical Surveys and Monographs. American Mathematical Society, Providence, RI, 2007.

[3] H. D'Angelo. Linear time-varying systems: Analysis and synthesis. (Allyn and Bacon Series in Electrical Engineering). Boston: Allyn and Bacon, Inc., 1970.

[4] B. Danhane, J. Lohéac, and M. Jungers. Characterizations of output controllability for LTI systems. Preprint, 2020.

[5] A. F. Filippov. Differential equations with discontinuous righthand sides, volume 18 of Mathematics and its Applications (Soviet Series). Kluwer Academic Publishers Group, Dordrecht, 1988. Translated from the Russian.

[6] E. Kreindler and P. E. Sarachik. On the concepts of controllability and observability of linear systems. IEEE Trans. Automatic Control, AC-9:129-136, 1964. (Correction to "On the concepts of controllability and observability of linear systems": p.118, 1965).

[7] P. E. Sarachik and G. M. Kranc. On optimal control of systems with multinorm constraints. IFAC Proceedings Volumes, 1(2):306-314, 1963.

[8] G. W. Stewart and J. G. Sun. Matrix perturbation theory. Computer Science and Scientific Computing. Academic Press, Inc., Boston, MA, 1990. 